\title{
HERMITIAN STRUCTURES ON HERMITIAN SYMMETRIC SPACES
}

\author{
F. Burstall* ${ }^{*}$ O. MušKarov**, G. Grantcharov** and J. Rawnsley* \\ Published as: J. Geom. Phys. 10 (1993) 245-249
}

\begin{abstract}
We show that an inner symmetric space with a compatible Hermitian structure is necessarily Hermitian symmetric, and the Hermitian structure must be invariant. This last result was known for some of the spaces of classical type and conjectured to be true for all compact Hermitian symmetric spaces in [2].
\end{abstract}

\section{INTRODUCTION}

In this note we show that a compact inner Riemannian symmetric space admits a complex structure compatible with the invariant metric precisely when the space is Hermitian symmetric and, further, that, in this case, the complex structure is invariant. This last result was conjectured to be true in [2] and proved for most of the classical Hermitian symmetric spaces with a case by case argument in unpublished work of D. Burns and the first author.

As an application of these results, we show that the only stable harmonic immersions of an compact inner Riemannian symmetric space into an irreducible compact Hermitian symmetric space are holomorphic maps of Hermitian symmetric spaces.

The first author would like to thank D. Burns for many stimulating and useful conversations on these topics.

\section{The Main Results}

Let $M$ be a $2 n$-dimensional manifold. We wish to study Hermitian (almost) complex structures on $M$ which we view as sections of the fibre bundle $\pi: J(M) \rightarrow M$ with fibre

$$
J_{x}(M)=\left\{j \in \operatorname{End}\left(T_{x} M\right): j^{2}=-1, j \text { is skew-symmetric }\right\} .
$$

This bundle is associated to the orthonormal frame bundle of $M$ with typical fibre $O(n) / U(n)$ which is a Hermitian symmetric space. Thus the vertical distribution $\mathcal{V}=$ ker $\pi_{*}$ inherits an almost complex structure $J^{\mathcal{V}}$. Moreover, the Levi-Civita connection $\nabla$ on $M$ induces a splitting

$$
T J(M)=\mathcal{V} \oplus \mathcal{H}
$$

with $\mathcal{H} \cong \pi^{-1} T M$ via $\pi_{*}$ so that $\mathcal{H}$ acquires a tautological complex structure $J^{\mathcal{H}}$ given by

$$
J_{j}^{\mathcal{H}}=j
$$

* Research partially supported by EEC contract SC1-0105-C.

** Research partially supported by Ministry of Education and Science of Bulgaria contract MM-54/91. 
Adding these gives an almost complex structure on $J(M)$ conventionally denoted by $J_{1}$.

In [6], Salamon shows that integrability of an almost Hermitian structure $J$ is equivalent to the holomorphicity in a certain sense of $J$ viewed as a map $M \rightarrow J(M)$. For this we first identify an almost Hermitian structure $J$ with its bundle $T_{J}^{+} \subset T M^{\mathbb{C}}$ of $(1,0)$-vectors. Then, specialising theorem 5.4 of [5] gives

Proposition 1.1. Let $J$ be an almost Hermitian structure on $M$. Then $J$ is holomorphic as a map $(M, J) \rightarrow\left(J(M), J_{1}\right)$ if and only if

$$
\nabla_{Z} C^{\infty}\left(T_{J}^{+}\right) \subset C^{\infty}\left(T_{J}^{+}\right)
$$

for all $Z \in C^{\infty}\left(T_{J}^{+}\right)$.

However, by lemma 1.2 and proposition 1.3 of [6], $J$ satisfies condition (1) if and only if it is integrable.

In fact we can say more. In [4], the Nijenhuis tensor $N^{J_{1}}$ of $J_{1}$ was calculated and, in particular, we have

Proposition 1.2. [4] Let $j \in J(M)$ with $(1,0)$-space $T^{+} \subset T_{\pi(j)} M^{\mathbb{C}}$. Let $R$ denote the Riemann curvature tensor of $\nabla$. Then the Nijenhuis tensor $N^{J_{1}}$ vanishes at $j$ if and only if

$$
R\left(T^{+}, T^{+}\right) T^{+} \subset T^{+} .
$$

In view of (1), we see that this condition is certainly satisfied for integrable $J$ and we may summarise our development in

Theorem 1.3. If $J$ is an almost Hermitian structure on the Riemannian manifold $(M, g)$ then $J$ is integrable if and only if it is holomorphic as a map $(M, J) \rightarrow\left(J(M), J_{1}\right)$. In this case the image of $J$ lies entirely in the zero-set of $N^{J_{1}}$.

In the case when $M$ is an inner symmetric space, the structure of the zero-set of $N^{J_{1}}$ was determined in [2]. The main results of that analysis are:

Theorem 1.4. [2] Let $M$ be an inner Riemannian symmetric space of compact type and $Z \subset J(M)$ the zero-set of $N^{J_{1}}$. Then

(i) each component of $Z$ is a flag manifold holomorphically embedded in $J(M)$;

(ii) if $M=M_{1} \times \cdots \times M_{k}$ is the de Rham decomposition of $M$ then $Z=Z_{1} \times \cdots \times Z_{k}$ where $Z_{i}$ is the zero-set of $N^{J_{1}}$ on $J\left(M_{i}\right)$.

From the first part of this theorem we immediately get:

Proposition 1.5. Let $M$ be an inner Riemannian symmetric space of compact type and $J$ a complex structure on $M$ compatible with the invariant metric. Then $J$ is Kählerian.

Proof. By theorem 1.3, $J$ is a holomorphic embedding of $M$ into $Z$ and thus, by theorem 1.4, a holomorphic embedding into a flag manifold. But flag manifolds admit Kähler metrics so we conclude that $M$ has a metric which is Kähler with respect to $J$.

As a consequence, we have: 
Theorem 1.6. Let $M$ be a compact inner symmetric space and $J$ be a complex structure on $M$ compatible with the invariant metric, then $M$ is Hermitian symmetric.

Proof. In view of theorem 1.4(ii), we have that $J$ respects the de Rham decomposition of $M$ so that, without loss of generality, we may assume that $M$ is irreducible. From proposition 1.5, we know that $M$ admits a Kähler metric and, since $M$ is compact, the corresponding Kähler form is not exact so that the real cohomology group $H^{2}(M ; \mathbb{R})$ is non-zero. But for an irreducible symmetric space, this can only happen if $M$ is Hermitian symmetric.

In particular, taking $M=S^{6}$, we recover the result of LeBrun [LeB] that $S^{6}$ admits no complex structure compatible with the round metric.

Let us now consider Hermitian structures on Hermitian symmetric spaces: in unpublished work, D. Burns has shown that the only Hermitian structure on $\mathbb{C} P^{n}$ for the Fubini-Study metric is the invariant one. We now extend this result to all Hermitian symmetric spaces of compact type.

Theorem 1.7. Let $M$ be a compact semisimple Hermitian symmetric space and $J$ a Hermitian structure for the invariant metric. Then $J$ is invariant.

Proof. We have already seen that $J$ must respect the de Rham decomposition, so we can assume that $M$ is irreducible. Let $K$ be an invariant complex structure and $g$ the invariant metric. We know from theorem 5.6 of [2] that $J$ and $K$ commute as endomorphisms of $T M$. Let $h$ be the Kähler metric for $J$ we get from proposition 1.5. The pairs $(g, K)$ and $(h, J)$ determine Kähler 2-forms

$$
\Omega(X, Y)=g(K X, Y) ; \quad \omega(X, Y)=h(J X, Y) .
$$

Both are closed forms and represent non-zero elements in the de Rham cohomology $H^{2}(M ; \mathbb{R})$. The second cohomology group of a compact irreducible Hermitian symmetric space is one-dimensional, so there is a non-zero real number $c$ with

$$
[\omega]=c[\Omega] .
$$

Thus there is a real 1 -form $\alpha$ with

$$
\omega=c \Omega+d \alpha
$$

Since $g$ is Hermitian with respect to $J$ then $\Omega$ like $\omega$ is type $(1,1)$ with respect to $J$, and hence so is $d \alpha$. Since $M$ is simply-connected and $J$ is Kähler, there is then a real-valued function $f$ on $M$ with $d \alpha=i \partial \bar{\partial} f$ (where $\partial$ is taken with respect to $J$ ).

Since $M$ is compact the smooth function $f$ will have a maximum at some point $x_{0}$ of $M$. At the maximum $x_{0}$ the $(1,1)$-form $i \partial \bar{\partial} f_{x_{0}}$ will be non-positive, so

$$
\omega_{x_{0}}(X, J X) \leq c \Omega_{x_{0}}(X, J X), \quad \forall X \in T_{x_{0}} M
$$

Translating in terms of the metrics we have

$$
h_{x_{0}}(X, X) \leq c g_{x_{0}}(K X, J X)
$$


On the other hand, since $J$ and $K$ commute their product $A$ is a symmetric endomorphism (with respect to $g$ ) with square the identity. Thus $A$ has only eigenvalues \pm 1 and so $T M$ splits into two smooth subbundles $T^{+}$where $J=K$, and $T^{-}$where $J=-K$. Suppose both subbundles are non-trivial, then at $x_{0}$ there is a non-zero vector $X^{+}$with $J X^{+}=K X^{+}$and a non-zero vector $X^{-}$with $J X^{-}=-K X^{-}$. Substituting into (2) we obtain

$$
c \geq \frac{h_{x_{0}}\left(X^{+}, X^{+}\right)}{g_{x_{0}}\left(X^{+}, X^{+}\right)}>0
$$

and

$$
c \leq-\frac{h_{x_{0}}\left(X^{-}, X^{-}\right)}{g_{x_{0}}\left(X^{-}, X^{-}\right)}<0
$$

which is a contradiction. Thus one of the two bundles $T^{+}, T^{-}$must vanish and hence $J= \pm K$.

Remark. In some classical cases, even stronger results are available: in unpublished work, D. Burns and F.E. Burstall have shown that if $M$ is a complex Grassmannian $G_{k}\left(\mathbb{C}^{k+n}\right), n, k>1$ or the Hermitian symmetric space $S p(n) / U(n)$, then $Z$ does not even admit continuous sections.

\section{APPLiCATION TO STABILITy OF HARMONIC MAPS}

In [1], the stability of harmonic maps into compact Hermitian symmetric spaces was studied and, in particular, the following result was proved:

Theorem 2.1. Let $\phi: M \rightarrow N$ be a stable harmonic immersion from a compact Riemannian manifold to a compact irreducible Hermitian symmetric space. Then $M$ admits a Hermitian complex structure with respect to which $\phi$ is holomorphic.

If we take $M$ to be an inner symmetric space of compact type and apply theorems 1.6 and 1.7, this gives:

Theorem 2.2. If $\phi: M \rightarrow N$ is a stable harmonic immersion from an inner Riemannian symmetric space of compact type to a compact irreducible Hermitian symmetric space, then $M$ is Hermitian symmetric and $\phi$ is holomorphic.

\section{REFERENCES}

[1] F. Burstall, D. Burns, P. de Bartolomeis and J. Rawnsley, Stability of harmonic maps of Kähler manifolds, J. Diff. Geom. 30 (1989), 253-290.

[2] F.E. Burstall and J.H. Rawnsley, Twistor theory for Riemannian symmetric spaces, Springer-Verlag, Heidelberg, 1990.

[2] C. LeBrun, Orthogonal complex structures on $S^{6}$, Proc. Amer. Math. Soc. 101 (1987), 136-138.

[4] N. O'Brian and J. Rawnsley, Twistor spaces, Annals of Global Analysis and Geometry 3 (1985), $29-58$.

[5] J.H. Rawnsley, $f$-structures, $f$-twistor spaces and harmonic maps, Geometry Seminar "Luigi Bianchi" II (E. Vesentini, ed.), Springer-Verlag, Heidelberg, 1985.

[6] S. Salamon, Harmonic and holomorphic maps, Geometry Seminar "Luigi Bianchi" II (E. Vesentini, ed.), Springer-Verlag, Heidelberg, 1985.

School of Mathematics, University of Bath, Bath BA2 7AY, United Kingdom 
E-mail address: feb@maths.bath.ac.uk

Institute of Mathematics, Bulgarian Academy of Sciences, Sofia 1113, Ul. "Acad. G. BoncheV", Bl 8, Bulgaria

Institute of Mathematics, Bulgarian Academy of Sciences, Sofia 1113, Ul. "Acad. G. Bonchev", Bl 8, Bulgaria

Mathematics Institute, University of Warwick, Coventry CV4 7AL, United Kingdom

E-mail address: jhr@maths.warwick.ac.uk 